\title{
Antimicrobial Susceptibility Profile of Gonococcal Isolates Obtained from Men Presenting with Urethral Discharge: Implication for National Syndromic Treatment guideline
}

\section{Surafel Fentaw Dinku ( sura4f@gmail.com )}

Ethiopian Public Health Institute https://orcid.org/0000-0002-8348-4965

\section{Rajiha Abubeker}

Ethiopian Public Health Institute

Nega Asamene

Ethiopian Public Health Institute

Meseret Assefa

Ethiopian Public Health Institute

\section{Eyasu Tigabu}

Ethiopian Public Health Institute

\section{Research article}

Keywords: Neisseria gonorrhoeae, Ciprofloxacin, Ceftriaxone MIC, Ethiopia

Posted Date: August 6th, 2019

DOI: https://doi.org/10.21203/rs.2.12433/v1

License: (c) (1) This work is licensed under a Creative Commons Attribution 4.0 International License.

Read Full License 


\section{Abstract}

Background: Neisseria gonorrhoeae (gonococcus) is one of etiologic agent for sexually transmitted diseases with high global significant public health importance. The treatment regimens for gonorrhea have changed frequently over the past few decades due to the organism's propensity for developing antibiotic resistance. This study was aimed to investigate quinolones, third-generation cephalosporins and other relevant antimicrobials susceptibility patterns of $\mathrm{N}$. gonorrhoeae so that the national treatment regimen could be revised based on the information generated from this study. Methods: Urethral discharge specimens were cultured on Modified Thayer Martín media and suspected gonococcal colonies were confirmed using Oxidase, Superoxol tests followed by commercial identification kit (API-NHR). Antimicrobial susceptibility testing was performed by Kirby-Bauer disc diffusion method using ciprofloxacin(5ug), ceftriaxone (30ug), cefixime(5ug), cefoxitin (30 ug), and spectinomycin (100 ug), on enriched GC agar. Minimum Inhibitory Concentration (MIC) was also done using concentration gradient strips (E-test) of the same antimicrobial agents. Results: The prevalence of gonococcal isolates from the current study was $69 \%$. Out of the 361 gonococcal isolates, close to $68 \%$ were fluoroquinolone non susceptible with $60 \%$ resistant and $7 \%$ intermediate status. However, there was no detection of ceftriaxone non susceptible gonococcal isolates. The isolates also showed reduced non susceptible to spectinomycin and cefoxitin. Conclusion: The prevalence of gonococcal isolates from the current study was high. There was a high level of fluoroquinolone resistance in gonococcal isolates recovered in this study. Key words: Neisseria gonorrhoeae, Ciprofloxacin, Ceftriaxone MIC, Ethiopia

\section{Background}

Neisseria gonorrhoeae (gonococcus), is one of etiologic agents for sexually transmitted diseases (STD), with global high prevalence. Transmission occurs by direct contact with secretions of infected mucosal surface, and the incubation period can range from 1 to 10 days (1). Gonorrhea, as well as any other STD, can work as a gateway to HIV and other infections with microorganisms (2). Public health control of gonorrhea requires treatment with appropriate antimicrobials, as well as generalized and targeted prevention efforts $(1,3,4,5)$.

Treatment regimens for gonorrhea have been changed frequently over the past few decades due to the organism's propensity for developing antibiotic resistance. Over the past few years, gonococcus have become less susceptible to previously used antibiotics such as sulfonamides or tetracycline. Until recently, quinolones and third-generation cephalosporins are alternative to treat gonococcal infections. However, the emergence and spread of gonococci resistant to the quinolone group of antibacterial was reported from different corners of the world $(4,6)$. This trend is concerning because no alternative antibiotic treatment options or combinations have been proven to be effective against the organism $(4,6,7,8,9,10)$.

The treatment for gonococcal infection in sub-Saharan Africa countries including Ethiopia was based on syndromic approach using single dose fluoroquinolone treatment. The basis for such a regimen was 
under the assumption that resistance to fluoroquinolones is considered to be low in Africa. However, with the occurrence of resistance to commonly prescribed antibiotics in both developed and developing countries, an updated knowledge of the prevailing susceptibility patterns of gonococci in Ethiopia is important for the proper selection and use of antimicrobial drugs as well as for the development of an appropriate prescription policy. This study was aimed to investigate the quinolones, third-generation cephalosporins and other related antimicrobials susceptibility patterns of $\mathrm{N}$. gonorrhoeae isolated from urethral discharge swab of patients seen in Addis Ababa health Centers.

\section{Methods}

\section{Study sites and design}

This study was conducted in Addis Ababa City Administration. The city is geographically located in central part of the country. A cross-sectional facility-based study was conducted in eight health centers of Addis Ababa. The selected health facilities were Arada, Tekalehaimanot, Addis Ketema, Kirkos, Kotebe, Akaki- Kaliti, Shiromeda and Kassanchis health centers. These health facilities were selected based on the assessment made purposively before this study on the flow of STI clients. There were eight study teams, one nurse and one overall study coordinator. Following training on study protocol, procedures, and research ethics, the study team stayed twelve months at the study site collecting samples.

\section{Source population, study participants}

The source populations were patients in the selected health facilities of Addis Ababa who were participated in the study. All urethral discharge specimens analyzed between August 2013 and August 2014 were included in this study.

\section{Laboratory methods}

Specimen Collection: Men presenting to the selected health facilities with urethral discharge syndrome were recruited in the study following their consent. A sterile cotton-tipped swab were used to obtain a swab specimens. Then sterile Dacron swabs tipped applicator were used to collect urethral secretions. The swabs were inoculated on in-house prepared Modified Thayer Martin Agar plates made of Gonococcal agar base supplemented with isovitalex (vitox); vancomycin, colistin, nystatin, and trimethoprim (VCNT); and synthetic hemoglobin (Oxoid and BBL). The inoculated plates were incubated on the site using candle jar and transported to the Ethiopian Public Health Institute (EPHI), Clinical Bacteriology and Mycology Reference Laboratory within the same day of collection. Swab was rolled onto a microscopy slide, labeled, heat fixed, placed in a slide box and sent to EPHI for Gram-stain analysis.

Culture and identification: In the clinical bacteriology laboratory, inoculated plates were incubated at $350 \mathrm{C}$ in carbon dioxide enriched environment (5-8 \% CO2) for 72 hours inspecting every day for the growth of small, translucent and non-pigmented colonies. Gram negative diplococcic convex, glistening, elevated, 
mucoid colony characteristics and oxidase, catalase and supercool $(30 \% \mathrm{H} 2 \mathrm{O} 2)$ positive were considered as probable N. gonorrhoeae and further confirmed by carbohydrate and enzymatic tests using API-NHR. Antimicrobial susceptibility testing was performed by Kirby-Bauer disc diffusion method using ciprofloxacin (5 ug) ceftriaxone (30 ug), cefixime (30 ug), cefoxitin (30 ug), and spectinomycin (100 ug), on enriched GC agar (Oxoid Ltd) plus 1\% BBL Isovitalex Enrichment. Minimum Inhibitory Concentration (MIC) was done using concentration gradient strips (E-test) of the same antibiotics. The range of inhibition zones and MIC for each type of antibiotic disk were interpreted according to Clinical Laboratory Standard Institute (CLSI) guideline (11). N. gonorrhoeae reference strain ATCC 49226 was used a positive control.

\section{Data extraction methods}

A structured check list was used to collect socio-demographic, clinical history, sexual behaviors, pro antibiotics taken and laboratory data such as the antibiotic susceptibility results.

All data were double entered to Cespro 8 soft by two individuals and data analysis were done using SPSS version 20.

\section{Operational definitions}

Non-susceptible N. gonorrhoeae isolates were defined as those that are not sensitive to the antibiotic tested for susceptibility, i.e., those isolates exhibiting resistance or intermediate resistance.

Dual non-susceptibility: non-susceptibility to any two of the antibiotics tested for susceptibility.

Multi Drug Non-susceptibility: Combined non-susceptibility to an injectable cephalosporin and any two of quinolones, penicillins or tetracyclines.

\section{Declaration}

\section{Ethics and Consent To Participate}

This study was ethically cleared by Scientific and Ethical Review Office (SERO) of Ethiopian Public Health Institute and the IRB of CDC-Atlanta.

At the enrollment visit, all men with urethral discharge (UD) were given written consent diagnosed according to the syndromic treatment guidelines currently approved in Ethiopia. Those who are eligible (> 18 years of age) and willing to participate in the study were asked using structured questionnaire for their demographic and behavioral data. All data were kept confidential anonymously. Brief counseling on the importance of adherence to STI medications, not having sex while taking medications, HIV/STI prevention, and recommendations to use condoms to reduce STI/HIV acquisition and transmission was also given. 


\section{Results}

A total of 599 urethral discharge specimens were collected from male patients presenting at eight health centers and the specimens were microbiologically analyzed at the clinical bacteriology reference laboratory of the Ethiopian Public Health Institute through bacterial culture and antimicrobial susceptibility testing following the standard operating procedures. The mean age of the study participants was 27 years $(S D+7.2)$, with all of them being male.

Of all the specimens analyzed, $415(69.3 \%)$ gonococcal isolates were identified (Table 1).

\section{Discussion}

The prevalence of gonococcus in this study was relatively higher than other studies conducted in Ethiopia $(12,13,14)$. The difference might be due to the nature of participants in the current study that all of them were males. The general notion is that naturally, males tend to be more symptomatic for gonococcal infection and hence can have increased level of health care seeking behavior which in turn make them more represented in the statistics (15).

In sub-Saharan Africa, gonococcal treatment practice is based on syndromic approach using single dose fluoroquinolone treatment. It is hypothesized that resistance to fluoroquinolones is low in Africa, but there has been limited systematic data collection and analysis to verify this notion. A multicounty antimicrobial resistance study on gonococcal strains isolated in 2004-2006, indicated low rates of fluoroquinolone resistance with $0 \%, 1.3$ and $4.0 \%$ in Central African Republic, Cameroon, and Madagascar, respectively (16). Similarly, study conducted in a Maputo and Mozambique, in 2005, suggested that there was no resistance to fluoroquinolone by gonococcal isolates (17).

In contrast, the findings from several other countries in sub-Saharan Africa suggested increasing levels of fluoroquinolone resistance in gonococcal isolates. According to a study done in South Africa, in 2004, 7\% of the gonococcal isolates from Pretoria region, $8 \%$ from Western Cape and 17\% from Johannesburg were found to be resistant to antibiotics from the class of fluoroquinolone. In addition, another study conducted in same country and the same study populations, in 2007 , indicated that; $27 \%$ of the gonococcal isolates from Cape Town and $32 \%$ from Johannesburg isolates were found to be resistant to ciprofloxacin (18). This represents 2.9 fold and 1.9 fold increases, respectively within a 3-year time period. Similarly, a 2-year prospective study carried out among STI patients, from 2004 to 2006, in Johannesburg indicated an increase in ciprofloxacin resistance from $13 \%$ in the first year to $26.3 \%$ in the second year (19). Study conducted in Kenya and Uganda also showed that gonococcal resistance level to fluoroquinolone has reached up to $53 \%$ and $83 \%$, respectively $(20,21)$.

The present study has revealed that $\mathrm{N}$. gonorrhoeae isolates recovered from this study have shown high level of resistance to commonly prescribed fluoroquinolone class of antibiotics in Ethiopia. This finding is in agreement with other studies which reported a resistance level of $53 \%$ and $83 \%$ in the East African region Kenya and Uganda. Reports from South Africa also indicate that the resistance level reached up to 
$32 \%(19,20,21,22)$. High proportion of quinolone resistance in this study might be due to prior treatment using ciprofloxacin as indicated in table 4. Gonococcal syndromic treatment using oral fluoroquinolone has become very problematic due to the emergence of high proportion of resistant isolates as witnessed from the current study. Non-susceptibility to ceftriaxone was not detected in all of the isolates tested during the study period. This finding is not in agreement with other studies conducted in different part of Ethiopia $(12,13,14)$. However, our finding was in agreement with other studies conducted elsewhere $(23,24,25,26,27)$. This might be due to exposure of participants for specific group of antimicrobial agents during the study period. As indicated in figure-1, the existence of certain segment of isolate population with MIC value close to the cutoff point may mean that there are concerns over the possibility of minority non-susceptible bacterial population to potentially replacing the susceptible majority ones. Therefore, investigating the molecular mechanism of resistance in these group of isolates may be imperative to fully understand the epidemiology (26).

Penicillinase test was done by chromogenic test and almost more than half of the isolates were positive for beta lactamase. Table 2, Table 3.. Most of the isolates in the current study were resistant to Benzyl penicillin even though the antibiotic is not used for the national gonococcal treatment algorithm. This finding from our study was in line with other studies $(24,25,27,28,29)$.

Formerly, the STI treatment practice in Ethiopia was based on the syndromic approach (treating individuals immediately for possible causes of STI syndromes based on symptom). However, because of the finding from this study, the national guideline for treatment has been changed. Besides this, the etiologic approach of diagnosis of gonorrhea is important at least for revisiting clients in order to identify non susceptible isolates for the current candidate of antimicrobial agents in practice.

\section{Conclusion}

In the current study, the proportion of $\mathrm{N}$. gonorrhoeae were found to be high. The most worrisome thing was that these gonococcal isolates were highly resistant to the new generation of antibiotics from fluoroquinolones (ciprofloxacin) which was indicated as a treatment of choice as per the previous guideline. The information generated from this study was used as in put to revise the national syndromic guideline for management of patients presenting with urethral discharge due to gonorrhea infection and therefore, ciprofloxacin was replaced by ceftriaxone which was found to be effective in terms the in vitro susceptibility result. In conclusion, the diagnostic approach, which is syndromic based management, needs to be periodically validated and modified based on determination of susceptibility patterns of $\mathrm{N}$. gonorrhoeae isolates.

\section{Declarations}

\section{Consent for Publication}

Not Applicable 
Availability of data and material

Data are available, we can share if necessary

\section{Conflict of Interest}

Authors declared no conflict of interest

\section{Funding}

This study was funded by CDC through the sexually transmitted infection surveillance program granted to Ethiopian Public Health Institute.

\section{Authors' contributions}

SF wrote the proposal, Laboratory work, analyzed the data and drafted the paper. RA, NA, and MA participated in data collection, entry and analysis. ET participated in the analysis and reviewed the manuscript. All authors participated in the preparation of the manuscript and approved the final manuscript.

\section{Acknowledgements}

We are grateful to CDC for financial and Technical support, Ethiopian Public Health Institute for permitting to conduct this study.

\section{Authors' details}

Ethiopian Public Health Institute, Bacterial, Parasitic and Zoonotic diseases research Directorate Clinical Bacteriology and Mycology Laboratory. P,O.Box 1242 Addis Ababa, Ethiopia.

\section{References}

1. WHO. Guidelines for the management of sexually transmitted infections.2003. Geneva: World Health Organization :1-91.

2. Sarwal S, Wong T, Sevigny C \& Lai-King NG. Increasing incidence of ciprofloxacin-resistant Neisseria gonorrhoeae infection in Canada. 2003. Canadian Medical Assocation, 168(7):872-873.

3. WHO .Guidelines for Sexually Transmitted Infections Surveillance UNAIDS/WHO Working Group on Global HIV/AIDS/STI Surveillance. 1999.Geneva: World Health Organization.

4. WHO.World Health Organization. Antimicrobial resistance in Neisseria gonorrhoeae. 2001. John Tapsall. WHO/CDS/CSR/DRS/2001.3

5. Workowski KA, Berman SM \& Douglas JM. Emerging antimicrobial resistance in Neisseria gonorrhoeae: urgent need to strengthen prevention strategies. 2008. Annals of Internal Medicine, 8 (148): 606-13. 
6. WHO. World Health Organization. Surveillance of antibiotic resistance in Neisseria gonorrhoeae in the WHO Western Pacific Region.2006.

7. Wi T, Lahra MM, Ndowa F, Bala M, Dillon J-AR, Ramon-Pardo P, et al. Antimicrobial resistance in Neisseria gonorrhoeae: Global surveillance and a call for international collaborative action. 2017. PLoS Med, 14(7).

8. Emily JW, Teodora Wi, John P. Surveillance for Antimicrobial Drug-Resistant Neisseria gonorrhoeae through the Enhanced Gonococcal Antimicrobial Surveillance Program. 2017. Emerging Infectious Diseases, www.cdc.gov/eid, 23, Supplement to December.

9. Magnus Unemo, \& William MS. Antimicrobial Resistance in Neisseria gonorrhoeae in the $21 \mathrm{st}$ Century: Past, Evolution, and Future. 2014. Clinical Microbiology Reviews, 27(3), 587-613.

10. Federal Democratic Republic of Ethiopia, Ministry of Health (MOH) National Guideline for the management of Sexually transmitted infections Using the Syndromic Approach. MOH.2006.

11. CLSI . Performance and presentation of cumulative Antimicrobial Susceptibility Test Data, Approved Guideline Fourth edition. M39-A4 document.2014. Clinical and Laboratory Standard Institute

12. Mengistu Hailemariam, Tamrat Abebe, Adane Mihret \& Tariku Lambiyo. Prevalence of Neisseria gonorrhea and their antimicrobial Susceptibility patterns among symptomatic women attending Gynecology outpatient department in Hawassa Refferal Hospital, Hawassa, Ethiopia. 2013. Ethiopian Journal of Health Science. 23 (1).

13. Addisu Gize Yeshanew \& Rozina Ambachew Geremew. Neisseria Gonorrhoae and their antimicrobial susceptibility patterns among symptomatic patients from Gondar town, North West Ethiopia. 2018. Antimicrobial Resistance and Infection Control. 7:85.

14. Tibebu M, Shibabaw A, Medhin G and Kassu A. Neisseria gonorrhoeae non-susceptible to cephalosporins and quinolones in Northwest Ethiopia.2013. BMC Infectious Diseases 13:415.

15. Seada Ali, Tsegaye Sewunet, Zewdineh Sahlemariam \& Gebre Kibru. Neisseria gonorrhoeae among suspects of sexually transmitted infection in Gambella hospital, Ethiopia: risk factors and drug resistance.2016. BMC Research Notes, 169:439

16. Cao V, Ratsima E, Van TD, Bercion R, Fonkoua MC, Richard V \&Talarmin A . Antimicrobial susceptibility of Neisseria gonorrhoeae strains isolated in 2004-2006 in Bangui, Central African Republic; Yaounde, Cameroon, Antananarivo, Madagascar; and Hi Chi minh Ville \& Nha Trang, Vietnam.2008. Sexually Transmitted Diseases,11: 941-5

17. Apalata T, Zimba TF, Sturm WA \& Moodley P. Antimicrobial susceptibility profile of Neisseria gonorrhoeae isolated from patients attending a STD facility In Maputo.2006. Sexually Transmitted Diseases, (36) 6: 341-343.

18. De Jongh M, Dangor Y, Adam A \& Hoosen AA. Gonococcal resistance: evolving from penicillin, tetracycline to the quinolones in South Africa - implications for treatment guidelines. 2007. International Journal of STD and AIDS,18: 697-699.

19. Lewis DA, Pillay C, Mohlamonyane O, Vezi A, Mbabela S, Mzaidume Y \& Radebe F. The burden of asymptomatic sexually transmitted infections among men in Carletonville, South Africa: implications 
for syndromic management. 2008. Sex Transmitted Infections, 84 371-76.

20. Hughes VJ, Matovu P, Bukenya G, Grosskurth J, \& Lewis, DA. High prevalence of ciprofloxacinresistant gonorrhea among female sex workers in kampala, Uganda (2008-2009). 2014. Sexually transmitted diseases, 41: 233-7.

21. Duncan S, Thiong'o AN, Macharia M, Wamuyu L, Mwarumba S, Mvera B,Smith AD \& Sanders EJ. High prevalence of quinolone resistance in Neisseria gonorrhoeae in coastal Kenya. 2011. Sex Transmitted Infections, 87(3):231.

22. Mehta D, Maclean I, Ndinya-Achola O, Moses S, Martin I, Ronald A, Agunda L, Murugu R, Bailey R, Melendez J \& Zenilman J. Emergence of quinolone resistance and cephalosporin MIC creep in Neisseria gonorrhoeae isolates from a cohort of young men in Kisumu, Kenya, 2002 to 2009. 2011. Antimicrobial Agents Chemotherapy, 55:3882-3888.

23. Susanne Buder, Sandra Dudareva, Klaus Jansen, Anna Loenenbach, Sergejs Nikisins, Andrea Sailer, Eva Guhl, Peter K. Kohl, Viviane Bremer \& Goronet. Study group Antimicrobial resistance of Neisseria gonorrhoeae in Germany: low levels of cephalosporin resistance, but high azithromycin resistance. 2018. BMC Infectious Diseases,18:44.

24. Clark M, Araneta P, Alain CJ et al. Antibiotic Susceptibility Monitoring of Neisseria gonorrhoeae in Bacolod City, Philippines. 2017. Tropical Medicine and Infectious Diseases, 2: 45.

25. Ahamed V N S, \& Shariff LB. A study on antimicrobial sensitivity pattern in Neisseria gonorrhoeae in a tertiary care hospital.2017. International Journal of Research in Medical Science, 5(9):3960-3965.

26. Ana Paula Ramalho da Costa-Lourenc,o, Késia Thaís Barros dos Santos, Beatriz Meurer Moreira, Sergio Eduardo Longo Fracalanzza \&Raquel Regina Bonelli. Antimicrobial resistance in Neisseria gonorrhoeae: history, molecular mechanisms and epidemiological aspects of an emerging global threat. 2017. Brazilian Journal of Microbiology, 4(8):617-628.

27. Sangeeta VK, Manju Bala, Syeeda AM, Sasikala G, Amit PN, Thorat R, et al. Antibiotic susceptibility pattern of Neisseria gonorrhoeae strains. 2018. isolated from five cities in India during 2013-2016. Journal of Medical Microbiology, 67:22-28.

28. Ranmini Kularatne, Venessa Maseko, Lindy Gumede \& Tendesayi Kufa. Trends in Neisseria gonorrhoeae Antimicrobial Resistance over a Ten-Year Surveillance Period, Johannesburg, South Africa, 2008-2017. 2018. Antibiotics, 7: 58.

29. Ranmini Kularatne, Venessa Maseko, Lindy Gumede, Frans Radebe \&Tendesayi Kufa-Chakezha. Neisseria gonorrhea Antimicrobial Resistance Surveillance in Guateng province, South Africa. 2016. Communicable Diseases Surveillance Bulletin, 14(3).

\section{Table}

Table 1: Gram stains and culture laboratory results of Urethral discharge specimen from patients 


\begin{tabular}{|c|c|c|c|}
\hline \multirow{2}{*}{$\begin{array}{l}\text { GC confirmation } \\
\text { Gram stain }\end{array}$} & Method Result & \multicolumn{2}{|c|}{ Frequency Percent } \\
\hline & Positive & 449 & $75 \%$ \\
\hline & Negativ & 150 & 25 \\
\hline Culture & Positive & 415 & $69 \%$ \\
\hline & Negativ & 184 & $31 \%$ \\
\hline Total & & 599 & 100 \\
\hline
\end{tabular}


Table 2: percent of Antimicrobial susceptibility pattern of gonococcal isolates from Urethral discharge patients $(\mathrm{N}=361)$

\begin{tabular}{|c|c|c|c|c|c|}
\hline Antimicrobial agent & Class & & Susceptibili & y profile & \\
\hline & & Resistant & Intermediate & Susceptible & $\begin{array}{l}\text { Non } \\
\text { susceptible }\end{array}$ \\
\hline Penicillin & Penicillins & 52.9 & & & \\
\hline Spectinomycin & Aminocyclitols & 3 & 1.1 & & \\
\hline Ciprofloxacin & Flouroquinolone & 60.2 & 7.1 & & \\
\hline Ceftriaxone & Cephalosposrin & - & & 100 & \\
\hline Cefixime & Cephalosposrin & & & 85 & 15 \\
\hline Cefoxitin & Cephalosporin & 1.1 & & & \\
\hline Azithromycin & Macrolides & 10 & & 90 & \\
\hline Cefoxitin + Ciprofloxacin & & . & & & \\
\hline Cefoxitin + Spectinomycin & & - & & & \\
\hline Ciprofloxacin + & & 0.6 & & & \\
\hline $\begin{array}{l}\text { Spectinomycin } \\
\text { Ciprofloxacin + penicillin }\end{array}$ & & 7 & & & \\
\hline
\end{tabular}

MIC Ranges for Resistance and Intermediate Susceptibility as defined by CLSI Guidelines:

Ciprofloxacin: Resistant $>1 \mathrm{ug} / \mathrm{mL}$, Intermediate $0.12-0.5 \mathrm{ug} / \mathrm{mL}$

Spectinomycin: Resistant $\geq 128 \mathrm{ug} / \mathrm{mL}$, Intermediate $64 \mathrm{ug} / \mathrm{mL}$

Ceftriaxone Sensitive $\leq 0.25$

Cefixime Sensitive $\leq 0.25$

Cefoxitin Resistant $\geq 8$, Intermediate 4

Azythromycin Sensitive $\leq 1$ 
Table 3. MIC Antimicrobial susceptibility pattern of gonococcal isolates from Urethral discharge patients $(\mathrm{N}=361)$

\begin{tabular}{|c|c|c|c|c|c|c|c|c|c|c|c|c|c|c|c|c|c|}
\hline \multicolumn{3}{|c|}{ nicilin } & \multicolumn{3}{|c|}{ Ciprofloxacin } & \multicolumn{3}{|c|}{ Spectinomycin } & \multicolumn{3}{|c|}{ Ceftriaxone } & \multicolumn{3}{|c|}{ Cefixime } & \multicolumn{3}{|c|}{ Azithromycin } \\
\hline & I & S & $\mathrm{R}$ & I & S & $\mathrm{R}$ & I & S & $\mathrm{R}$ & I & S & $\mathrm{R}$ & I & S & $\mathrm{R}$ & & S \\
\hline & - & 170 & - & - & 78 & - & - & - & - & - & 245 & - & - & 323 & - & - & - \\
\hline & - & - & - & - & 41 & - & - & - & - & - & 47 & - & - & - & - & - & 156 \\
\hline & - & - & - & - & - & - & - & - & - & - & 12 & - & - & - & - & - & \\
\hline & - & - & - & - & - & - & - & - & - & - & 53 & - & - & - & - & - & 140 \\
\hline & - & - & - & 23 & - & - & - & - & - & - & 4 & - & - & - & - & & 4 \\
\hline & - & - & - & - & - & - & - & - & - & - & - & - & - & - & - & - & 46 \\
\hline & 20 & - & 24 & - & - & - & - & 127 & - & - & - & - & - & - & - & - & - \\
\hline & - & - & - & - & - & - & - & 39 & - & - & - & - & - & - & 8 & - & - \\
\hline 1 & - & - & 37 & - & - & - & - & 43 & - & - & - & - & - & - & 6 & - & - \\
\hline & - & - & - & - & - & - & - & 57 & - & - & - & - & - & - & 1 & - & - \\
\hline & - & - & - & - & - & - & - & 38 & - & - & - & - & - & - & - & - & - \\
\hline & - & - & 158 & - & - & 11 & 4 & 42 & - & - & - & - & - & - & - & - & - \\
\hline 1 & 20 & 170 & 219 & 23 & 119 & 11 & 4 & 346 & - & - & 361 & - & - & 323 & 15 & - & 346 \\
\hline
\end{tabular}

Table 4: Medical treatment history of patients with Urethral discharge from health centers in Addis Ababa, August 2013- August 2014 


\begin{tabular}{llll}
\hline Medication history & Response & Number & Percent(\%) \\
\hline Taking medication & Yes & 123 & 20.5 \\
& No & 476 & 79.5 \\
& Total & 599 & 100 \\
Know the type of medication & Yes & 107 & 87 \\
& No & 16 & 13 \\
& Total & 123 & 100 \\
Ciprofloxacin & Yes & 91 & 85 \\
& No & 16 & 15 \\
Doxycycline & Total & 107 & 100 \\
& Yes & 93 & 86.9 \\
& No & 14 & 13.1 \\
Metronidazole & Total & 107 & 100 \\
& Yes & 12 & 11.2 \\
& No & 95 & 88.8 \\
& Total & 107 & 100 \\
\hline
\end{tabular}

Figures

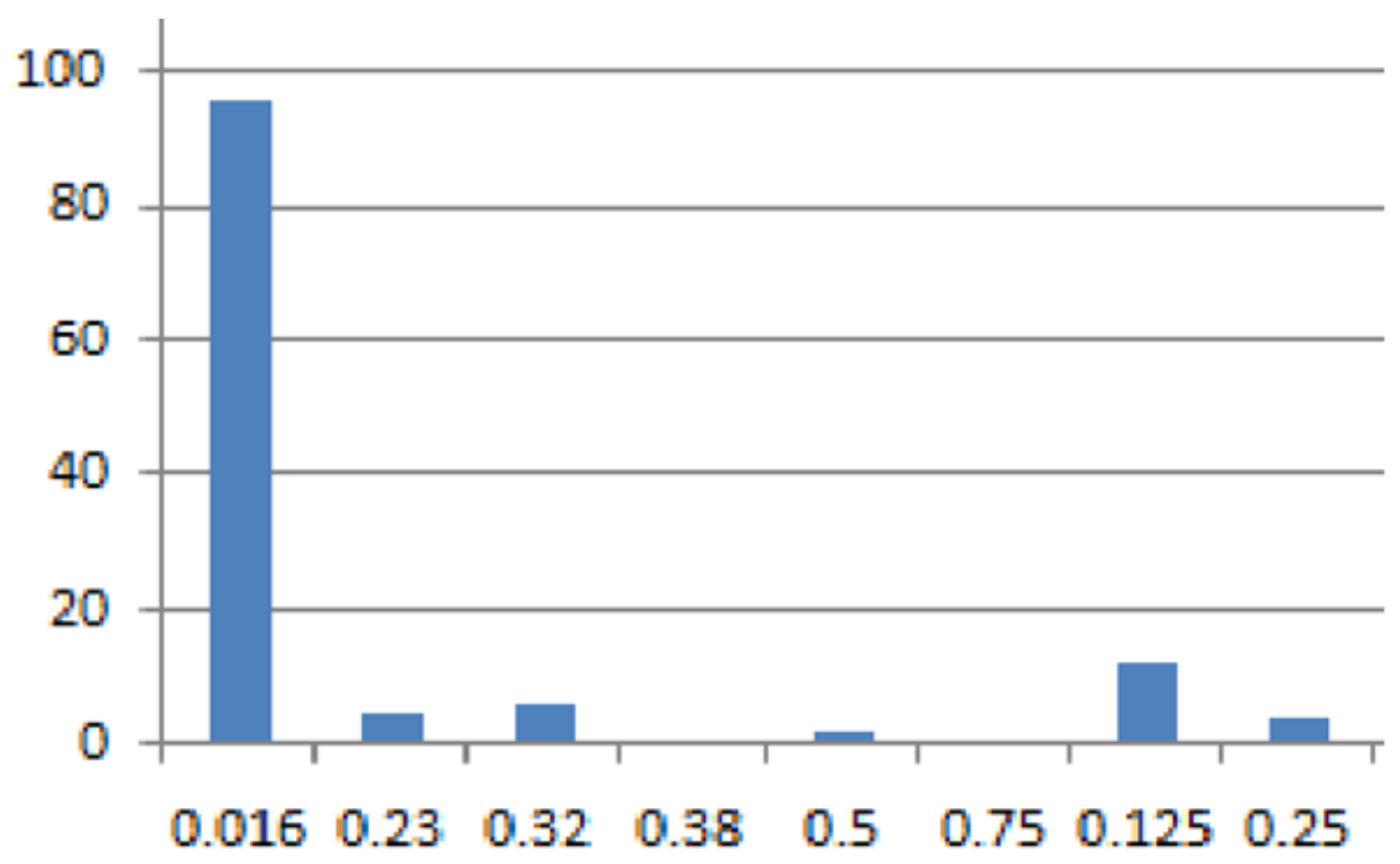


Figure 1

Minimum inhibitory concentration of ceftriaxone against N. gonorrhea

\section{Supplementary Files}

This is a list of supplementary files associated with this preprint. Click to download.

- gonococcus.rar 